\title{
Research on CNC Machining Process and Programming Technology of Complex Parts Based on PowerMILL
}

\author{
Cao Shuling ${ }^{1}$ and Guan Jinbiao ${ }^{2}$
}

\author{
(1.NanChang Institute of Science and Technology, Nanchang,330108; Jiangxi Engineering \\ Laboratory of Digital Manufacturing for Automobile Parts; 2. AnHui QuanChai Engine CO.,LTD)
}

\section{Keywords: PowerMILL; CAD/CAM; Complex Parts; Numerical Control Processing; Technology}

\begin{abstract}
CNC machining of complex parts refers to cutting simulation based on CAD/CAM and processing of post-programs. Among them, the cutting simulation is mainly used in NC program inspection, and the subsequent program processing is mainly the adjustment and processing of the tool path, which is a supplement to the CAD/CAM programming function. In order to improve the quality and processing efficiency of complex surface NC machining, the article studied the application of cutting simulation and its subsequent program processing in product NC machining based on its core technology, namely CAD/CAM.
\end{abstract}

\section{Introduction}

At present, China's social economy is steadily moving forward. Our country has been widely used and rapidly developed in the field of computer technology. Computer technology has also been widely used in numerical control processing and programming. CNC technology and CNC-related equipment are important material foundations in the modernization of manufacturing industry. Intelligentization, openness, and networking have become the main trends in the development of modern numerical control systems. This paper mainly deals with the numerical control of complex surfaces and programming. Technology analysis and discussion.

\section{Introduction to PowerMILL}

PowerMILL has a complete processing plan, no need for human intervention in the preparation of the processing model, no requirement for the operator, the programmer can easily complete the work, and focus on other important things. At the same time, it is also a representative and faster growth processing software of CAM software technology.

PowerMILL can accept three-dimensional computer models generated by different software systems, allowing manufacturers to use many different CAD systems without having to repeat investment.

PowerMILL is an independently operated, most intelligent 3D complex body processing CAM system. The CAM system is separated from the CAD and integrated in the network. It is more adaptable to engineering requirements and represents the latest development direction of CAM technology. Compared with most of today's curved CAM systems, there are unparalleled advantages.

In the actual production process, the design (CAD) and manufacturing (CAM) locations are different, and the emphasis is not the same. Most surface CAM systems today are functionally and structurally hybrid CAD/CAM systems. Can not meet the structural requirements of design and manufacturing separation. PowerMILL realized the separation of CAD systems and realized system integration in the network, which is more in line with the natural requirements of the production process.

The PowerMILL system operation process fully complies with the engineering concept of CNC machining. Solid model automatic processing, to achieve the coarse, fine, clear root processing programming automation. The degree of difficulty in programming is not related to the complexity of the part. As long as the CAM operator has knowledge of the processing technology, only 2-3 
days of professional technical training can be used to numerically program very complex molds.

PowerMILL's Batchmill function realizes automatic programming according to the process file and lays the foundation for future integrated integration of CAD/CAPP/CAM.

\section{Analysis of Complex Parts Machining Process}

(1) Choose equipment

The X52K milling machine and M1420 grinding machine are selected based on the shape and material of the parts to be machined.

(2) Determine the part positioning reference and clamping method

In order to ensure the coaxiality and other positional accuracy of the cylindrical surfaces of the complex parts, the reference axis should be selected as the reference for the semi-finisher, finisher, and grinding, and the center hole should be drilled at both ends of the shaft and clamped with two tips. The relative size, position accuracy and roughness of the center hole at both ends are important factors affecting the machining accuracy. Therefore, the workpiece must be arranged after grinding and other heat treatment. In order to ensure the rigidity of the work piece when the roughing machine is used, a clamping method is often used for the clamping of the work piece. The left end adopts a three-jaw self-centering chuck for clamping and the right end adopts a movable top support top.

(3) Formulate processing plan

The processing route is determined according to the processing principle of first, second, first and second, then rough machining with a fixed cycle command, finishing, and threading.

(4) Tool and location number

Roughing and semi-finishing vehicles use the same $90^{\circ}$ alloy turning tool and they are installed on the No. 1 tool post of the tool holder and set as the reference tool. The car groove uses two slotted knives, one main cutting edge is $2 \mathrm{~mm}$ wide, and the other main cutting edge is $1.36 \mathrm{~mm}$ wide. The tool point is taken at the left tip. They are mounted on the No. 2 and No. 3 tool positions of the tool holder. The thread of the thread is $60^{\circ}$ external thread turning tool, and the tool nose angle is $59^{\circ} 30^{\prime}$. It is mounted on the 4th tool post of the tool holder. The tool position deviation value of each tool is measured by the test-cutting knife method and is run in the program. Before entering the CNC system.

(5) Write a program

The use of complex parts for programming design, in the thread turning programming should pay attention to, CNC lathe spindle must be installed with a pulse encoder to determine the actual spindle speed, in order to achieve a spindle rotation tool feed a thread lead synchronous movement, from the thread coarse From the car to the finishing car, the spindle speed must be kept constant. The complex part has a relatively simple structure with threads, chamfers, keyways, and undercuts. Since the part is mainly used for fitting, the clearance must also be taken into account. Tolerances, form tolerances, etc.

\section{CNC Machining Programming for Complex Parts}

Analytical complex parts have low-level implicit function expressions and high-level parameter expressions, which means that all curves and surfaces can be uniformly represented by parameter expressions (that is, non-uniform B-spline NURBS in ACIS). The topological geometry represents the connection relationship between the different elements of the constituent geometry. The topological structure is decomposed hierarchically into the following objects: BODY, LUMP, SHELL, SUSHELL, FACE, LOOP, wIRE, COEDGE, and EDGE o Relationship between topological objects, as shown in Figure 1. 


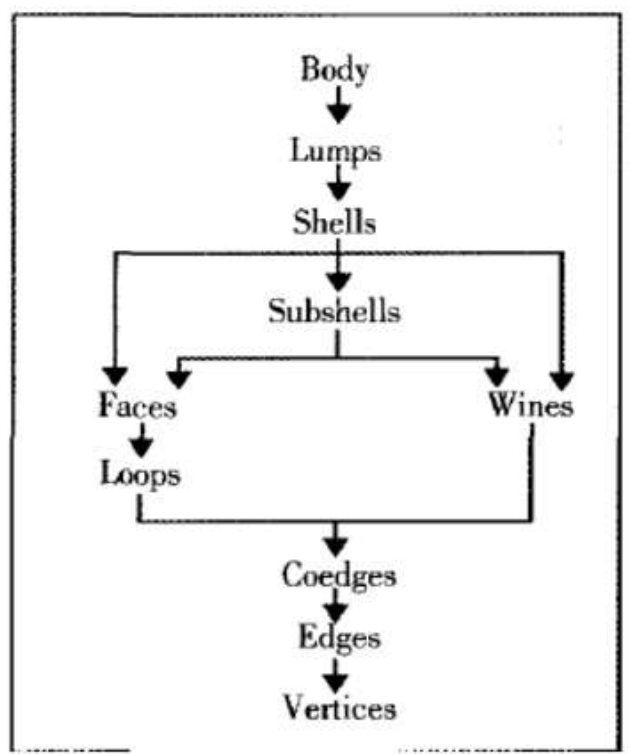

Figure 1 Relation ship between topological objects

The automatic generation of interference-free tool trajectories for complex part surface finishing is realized. The example verification shows that the method is simple, stable and reliable, the tool path is continuous and efficient, and the machining accuracy of the machined parts can be guaranteed while improving the machining efficiency. For a complex part surface, we can obtain the correlation of each surface through the topological relationship; we can obtain the geometric information of each surface by accessing the geometry information, such as the spatial coordinates of a point on the surface, the method of loss, and the direction along a certain direction. Order and second derivative and so on. There is no need to use different evaluation methods for different types of surfaces, which greatly simplifies the algorithm.

The problems occurred in the NC programming of complex parts using CAPP / CAM technology, respectively using the priority classification search strategy and processing template technology, the similarity in the CAPP / CAM mining and processing; and through the use of UG secondary development Technology and SQL server database technology developed a numerically-controlled automatic programming system to utilize similarities mined from CAPP/CAM to realize the customization of processing templates on the UG platform, matching with the CAPP process, and automatic NC programming; application examples verified The feasibility and practicality of the system can greatly improve the efficiency and programming quality of numerical control programming for complex parts.

\section{Summary}

Design must not only be based on manufacturing, but also be innovative, but we must learn to inherit. The entire process from the drawing of parts to the preparation of part processing programs and the production of control media is called programming. The programming is very important in industrial production. Therefore, it is necessary to compile the processing procedures of the parts. The parts are processed by milling machines, and attention must be paid to program preparation. At the same time, it is necessary to meet the programming features of the $\mathrm{CNC}$ milling machine, and at the same time pay attention to the use of some instructions, which is not the same as the CNC lathe.

\section{Acknowledgement}

Project funding: Project at Nanchang institute of science and technology (No.SZZX-17-08)

\section{References}

[1] Luo S. Research and Application in NC Machining Based on UG[J]. Hunan Agricultural 
Machinery, 2008.

[2]Li Y G, Fang T L, Cheng S J, et al. Research on Feature-Based Rapid Programming for Aircraft NC Parts[J]. Applied Mechanics \& Materials, 2008, 10-12:682-687.

[3]Guo Y. Machining Technology of Complex Surface Machining Based on Research with Pro/e[J]. Science \& Technology Information, 2012.

[4]Gao H. Research on on-machine Measurement Technology based on PowerINSPECT[J]. New Technology \& New Process, 2013.

[5]Da-Peng F U, Yan-Li M A, Zhu Y L. Five-axis NC machining technology research based on cimatron E8.5 marine propellers[J]. Heavy Machinery, 2013.

[6]Chen Z Y, Wang X E, Yang M. Complex Parts Design Based on Macro Programming[J]. Applied Mechanics \& Materials, 2014, 635-637:1386-1389.

[7]Wang W K, Huang J. Research on Five-axis Complex Surfaces Machining Based on Power MILL[J]. Journal of Nanjing Institute of Industry Technology, 2015.

[8]Han Z, Sheng M, Liu B. Research about Using Flat-bottomed Cutter on Five-axis NC Programming Technology Based on CATIA[J]. Machine Tool \& Hydraulics, 2016.

[9]Ye X L. Based on Solidworks and Mastercam Combined for Rub a Drum of Four-Axis NC Machining Programming Research[J]. Applied Mechanics \& Materials, 2014, 599-601:225-229.

[10]Du X, Liu S. Research on Group Technology in NC Automatic Programming System for Complex Parts[J]. Journal of Henan Science \& Technology, 2016.

[11]Jiao H. Research on NC Turning Technique based on Finishing Machining[J]. New Technology \& New Process, 2013.

[12]Wang H. Research and application of CAPP technology in NC machining based on drawing die[J]. Jiangsu Science \& Technology Information, 2017. 